\title{
Fabrication of Carbon Nanotubes for High-Performance Scanning Probe Microscopy
}

\author{
Ian Thomas Clark and Masamichi Yoshimura \\ Surface Science Laboratory, Toyota Technological Institute, 2-12-1 Hisakata, Tempaku, \\ Nagoya \\ Japan
}

\section{Introduction}

The benefits of using carbon nanotubes (CNTs) as probes for scanning probe microscopy (SPM) have been recognized for many years. ${ }^{1}$ Since the initial report on the fabrication and use of CNT SPM probes, many accounts of the fabrication of CNT SPM probes, and demonstrations of the superior performance of these probes have been published. Significant progress has been made since the initial studies by Dai et al.,1, 2 although a method for fabricating CNT SPM probes that fully exploits the desirable properties of the $\mathrm{CNT}$ and is truly reproducible and cost-effective remains elusive. In the present work, we detail the specific properties of CNTs that make them appropriate for various SPM methods, and review methods for the fabrication of CNT SPM probes. Our review of fabrications methods includes a review of methods in the literature, as well as recent, previously unpublished methods developed in our laboratory. The goal of this work is to provide a concise and up-to-date guide to aid researchers in the further development of CNT SPM probes.

SPM now encompasses several tens of distinct techniques, and is arguably the most widely used method to obtain real-space information on surfaces and solid-fluid interfaces; in the past several years SPM has been applied even to fluid-fluid interfaces, such as imaging living cells in buffer solution. ${ }^{3}$ The basic block diagram of a general SPM instrument is presented in Fig. 1. In all variants of SPM, a probe is brought close to the surface or interface of interest, and rastered across the surface/interface while one or more interactions between the probe and the surface/interface is transduced and recorded. In many, but not all cases one of the transduced interactions is used in a feedback loop to maintain a constant distance between the probe and the surface/interface. Scanning tunneling microscopy (STM), the first SPM method reported, ${ }^{4}$ transduces the quantum mechanical tunnel current from a sharp metal probe biased relative to a sample. Since the introduction of STM the list of SPM methods has grown dramatically. A non-exhaustive list of commonly used SPM methods includes several varieties of atomic force microscopy (AFM), which map surface/interface morphology by transducing the short-range van der Waals and capillary forces between the surface/interface and probe, ${ }^{5-8}$ magnetic force microscopy (MFM), which maps the stray magnetic field above a surface/interface by transducing the force on a magnetic probe due to a magnetic or current-carrying sample,,-11 electrostatic force microscopy (EFM), which maps the electrostatic field above a surface/interface by transducing the force on a 
conductive probe biased with a DC voltage relative to the sample, ${ }^{12,13}$ and Kelvin force microscopy (KFM), which maps the contact potential by transducing the AC component of the capacitive force on a conductive probe biased with both AC and DC components relative to the sample.14, 15 The demands on the probe vary widely from method to method; however, as we will describe here, for many of these methods, carbon nanotubes make an excellent probe material.

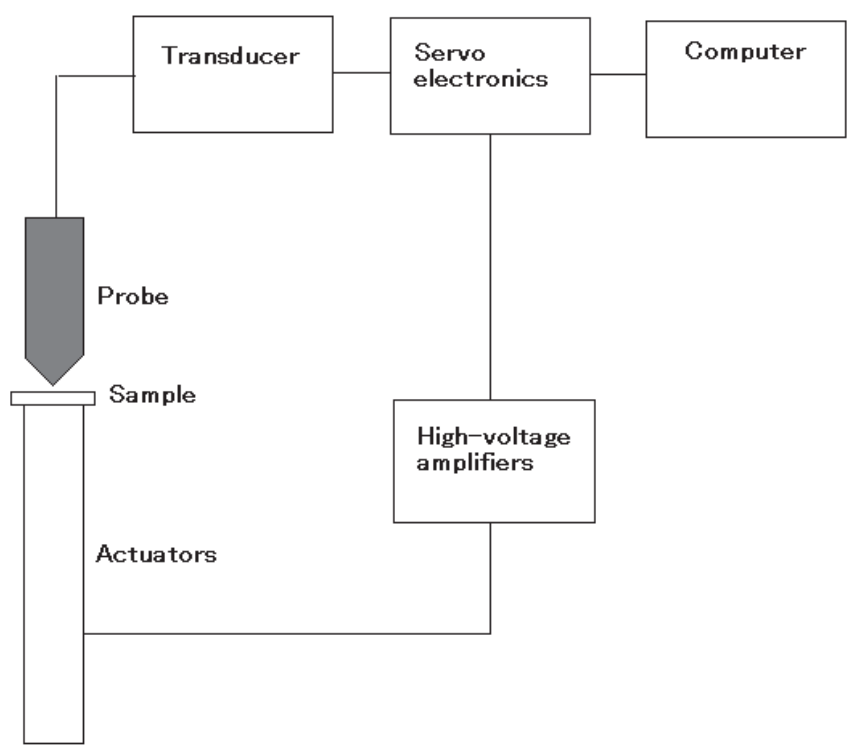

Fig. 1. Block diagram of a generic SPM instrument. Servo electronics are frequently, but not always used to maintain a constant sample-probe distance.

Just as "SPM" describes a family of related microcopy methods rather than a single method, "CNT" describes a family of related nanostructures rather than a specific nanostructure; CNTs vary widely in number of walls and diameter. Single-wall CNTs (SWNTs) and individual walls of multi-wall CNTs (MWCNTs) also differ in helicity - the manner in which the graphene lattice is wrapped to form the cylindrical CNT wall. The electronic and mechanical properties of a CNT depend, sometimes strongly, on these geometric attributes. ${ }^{16}$ CNTs with a certain diameter and number of walls can be grown by choosing an appropriate growth method from the myriad of published CNT growth methods. ${ }^{17}$ With modern purification methods, even SWNTs of a specific helicity can be obtained. ${ }^{18}$ Further, catalytic chemical vapor deposition (CVD) growth methods can be used to make CNTs encapsulating metal particles and nanowires, ${ }^{19-21}$ and methods of plating the exterior of CNTs with metals and electrically insulating layers have also been published;22, 23 these metal/CNT and insulator/CNT hybrid structures naturally have different electronic, mechanical and magnetic properties. Developers of CNT SPM probes therefore have a great deal of control over the properties of the CNTs used in probe manufacture. In an effort to guide CNT SPM probe developers to the optimal CNT variety for their specific SPM application, the following three sections relate CNT attributes to 
electronic/magnetic/mechanical properties, and further relate those properties to the design requirements of probes for various varieties of SPM.

\section{The benefits of CNTs for SPM applications}

\subsection{Geometric considerations and probe convolution}

It was recognized many years ago that the shape of CNTs is almost ideal for use as a SPM probe. Generally, the goal of SPM is to map some property of a surface/interface in real space; however, the image acquired by SPM is not a map of that property of the sample, but rather a convolution of the properties of the sample and the SPM probe. This is illustrated with an idealized contact-mode AFM experiment in Fig. 2: as the pyramidal probe of Fig. 2 (a) is rastered over the rectangular protrusion, it traces a path that is sloped at the angle of the pyramidal probe, rather than vertical like the actual profile of the protrusion. The SPM "image" reflects both the shape of the surface and the shape of the probe. As is illustrated in Fig. 2(b), the pseudo-one-dimensional nature of CNTs can minimize erroneous features in the SPM image due to probe convolution.

\section{(a) Standard} SPM Probe
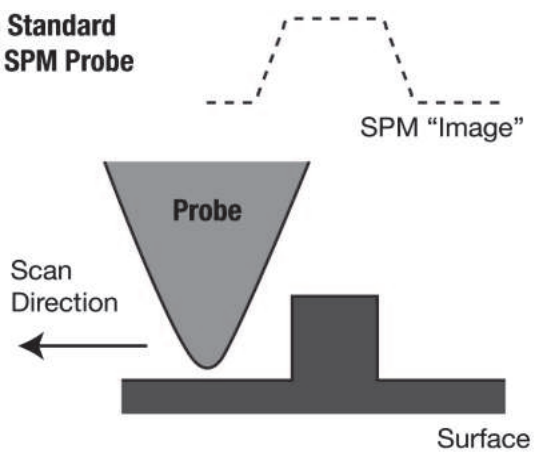

(b) CNT SPM Probe
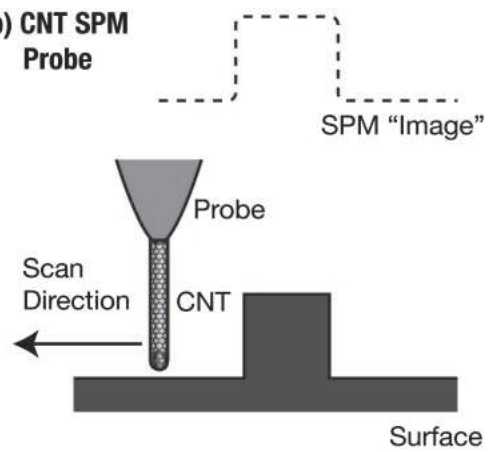

Fig. 2. Idealized example of tip convolution: a standard SPM probe (a) and a CNT SPM probe (b) going over the same square surface feature. Dashed line indicates the path of the probe as it is rastered over the surface. This path is recorded as the SPM image.

In SPM methods relying on long-range forces (e.g. MFM, EFM, etc.), probe convolution is more complicated and in many cases can lead to significantly greater difficulty in interpreting the resulting SPM image. Here, we use MFM as an exemplar to demonstrate the potential of CNT probes for SPM methods relying on long-range forces.

To a first approximation, the magnetic interaction energy of a probe with a magnetization $\mathbf{M}_{\text {probe }}(\mathbf{r})$ at the point $\mathbf{r}$ with the magnetic field induced by the sample, $\mathbf{H}_{\text {sample }}(\mathbf{r})$ can be expressed as

$$
E=\int_{\text {probe volume }} \mathbf{H}_{\text {sample }}(\mathbf{r}) \cdot \mathbf{M}_{\text {probe }}(\mathbf{r}) d V,
$$

where the integral is taken over the volume of the probe. As in AFM, the probe is mounted on a cantilever driven at a frequency near its resonant frequency. The force gradient due to the magnetic interaction (i.e. the second $z$ derivative of interaction energy) weakens or enhances the restoring force of the cantilever. The resulting shift in resonance frequency is detected as a shift in the relative phases of the driving force and the motion of the cantilever. 
In practice the cantilever is typically kept a few tens of nanometers above the surface during MFM phase mapping to prevent interference of the stronger but shorter-range van der Waals and capillary forces.

Several factors complicate analysis of these MFM phase maps: first, magnetic field decay above the sample depends on the lateral wavelength of the magnetization field of the sample. More rigorously, a sample with a magnetization field

$$
\mathbf{M}=\mathbf{M}_{0} \sin (2 \pi x / l),
$$

where $x$ is in the plane of the sample surface, will induce a magnetic field that decays exponentially away from the surface with the characteristic decay length $l$. Viz,

$$
\mathbf{H}_{\text {sample }} \propto \exp \left(-z^{2} / l\right) .
$$

If Eq. 2 is considered to be a Fourier component of an arbitrary magnetization field, it is clear from Eq. 3 that lateral variations in the magnetic field above the sample are effectively a low-pass-filtered version of lateral variations in the magnetization field since smallerwavelength components of the magnetic field decay more rapidly away from the surface.

A second complicating factor can be seen from Eq. 1: the phase shift mapped in MFM does not arise from a de facto surface-surface interaction as it does in AFM, but rather the interaction between the stray magnetic field and the entire volume of the magnetic material of the probe; the shape of the entire probe, rather than just the probe apex, contributes to the convolution. To illustrate, we compare a typical commercial MFM probe, which is made by depositing a few-nanometer layer of magnetic material over an approximately pyramidal silicon probe, as shown in Fig. 3(a), with a CNT-encapsulated magnetic nanoparticle MFM

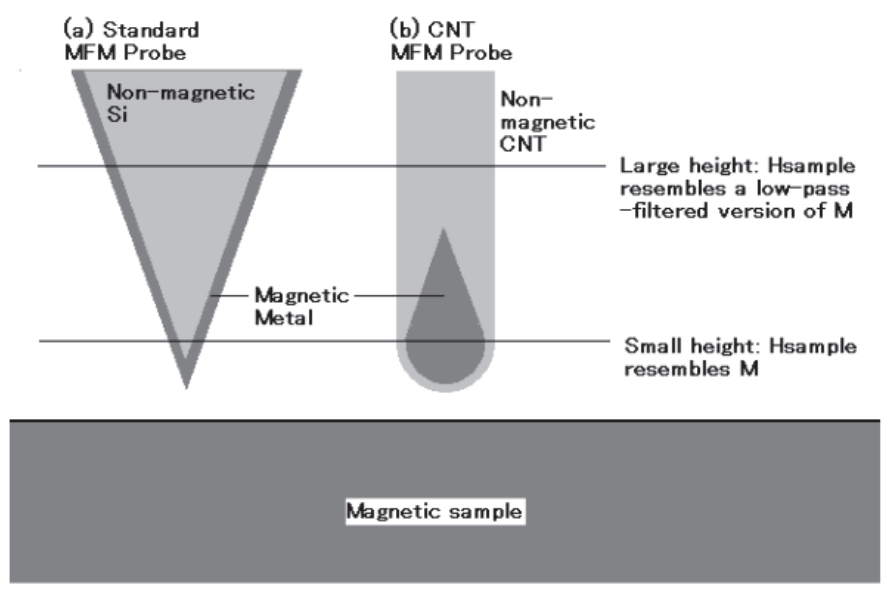

Fig. 3. Cartoon of a (a) standard MFM probe composed of a layer magnetic material over a pyramidal, non-magnetic probe, and (b) a CNT MFM probe composed of a magnetic nanoparticle encased in the tip of a CNT interacting with the stray magnetic field induced by a magnetic sample. Note that the CNT SPM probe has a larger fraction of its magnetic material closer to the sample surface, where the stray magnetic field is a more faithful representation of the samples magnetization field. 
probe, like those recently reported by Tanaka et al. (fabrication method discussed below). ${ }^{24}$ The magnetic catalyst particle used to grow these CNT probes is in the tip of the CNT and has a characteristic "carrot"-shape as illustrated in Fig. 3(b). The amount of magnetic material in a probe between in a differentially thin slice between $z$ and $z+d z$ increases monotonically with increasing $z$ (i.e. increasing distance from the sample surface) from the tip of the probe for the standard probe. For the CNT probe, this value increases rapidly in the first few nanometers from the tip, but then decreases with increasing $z$, going to zero at tens to a few hundreds of nanometers from the CNT tip. Since this differentially thin slice of the probe between $z$ and $z+d z$ interacts with the sample-induced magnetic field at $z$, and the low pass filtering effect of the sample-induced magnetic field increases with increasing $z$, it is clear that less material at larger $z$ is preferential for high-resolution MFM imaging. Unlike AFM where a minimal lateral dimension of the probe is desirable, in MFM both a minimum lateral and vertical dimension are desirable. Since a finite volume of magnetic material is needed in the probe, one must in general balance the criteria of low lateral and low vertical dimension. In light of this analysis, due largely to its small effective vertical dimension, CNT MFM probes are preferable to standard MFM probes. Indeed, we have found that even fairly wide CNT MFM probes (tube radius as large as $60 \mathrm{~nm}$ ) provide significantly higher resolution MFM images than significantly sharper (tip radius of curvature of approximately $15 \mathrm{~nm}$ ) standard MFM probes. ${ }^{24,} 25$ While MFM is the only example of its kind provided here CNT SPM probes have been proposed for EFM and other SPM methods relying on longrange forces based on similar geometric arguments.

\subsection{Mechanical considerations}

The mechanical properties of CNTs have been celebrated for many years and have been the subject of hundreds of experimental and theoretical investigations, as reviewed most recently by Wang et al, ${ }^{26}$ and Shokrieh et al. ${ }^{27}$ As is often cited, the Young's modulus and tensile strength of CNTs are five and 120 times greater, respectively, than those of 304 stainless steel. Although these simple numbers indicate that CNTs are in general mechanically robust, the mechanical properties of CNTs depend on their diameter, number of walls, and aspect ratio. To understand how the mechanical properties of CNTs are beneficial in SPM applications as well as to select the optimal CNT length, diameter, and number of walls for a given SPM method, it is important to consider the forces applied to the SPM probe during operation.

Tip "crashes" are a common problem in almost all forms of SPM; mistakes made in approaching the probe to the sample, environmental vibrations large enough to transmit through the instrument's vibration isolation systems, or rastering the probe across a surface feature too steep for the servo circuit to avoid, or too large for the servo actuators to overcome can all result in a probe-sample collision that applies extremely large transient stresses to the probe apex breaking brittle probe materials or plastically deforming metal probe materials. While fracture and plastic deformations of CNTs have been observed in experimental ${ }^{28-30}$ and theoretical ${ }^{31,32}$ investigations, the strains leading to fracture or plastic deformation are unlikely to be encountered in even the most violent tip crash. Unlike more common probe materials, both SWNTs and MWCNTs can be deformed to a remarkable extent, and regain their original shape when the stress is relieved. ${ }^{33-38}$ In our experience, $\mathrm{CNT}$ probe destruction during tip crash is generally the result of failure of the CNT/probe 
junction, rather than failure of the CNT itself. To minimize stress on the CNT/probe junction, the thinnest and longest and therefore most compliant CNT compatible with the particular SPM application should be used. As described above, long, thin CNTs also minimize tip convolution for most SPM methods.

In noncontact (NC)- and TappingMode ${ }^{\mathrm{TM}}(\mathrm{TM})-\mathrm{AFM}$, and most methods based on NC- and TM-AFM (e.g. EFM, MFM, KFM, etc.), the forces applied to the probe under normal operation are extremely small. Thus, thin, high-aspect-ratio CNTs can be used for probes. In practice, above a certain aspect ratio the resolution is found to decrease with increasing CNT length, ${ }^{39}$ an effect that most authors attribute to thermally excited vibrations in the CNT;39, 40 however, the extremely high elastic modulus of CNTs results in a very low susceptibility to thermal vibrations, and excellent resolution has been reported for CNT SPM probes with aspect ratios of more than 50. Interestingly, Carbon Nanotube SPM Probes commercially available from NanoSensors ${ }^{\mathrm{TM}}$ use 1.2- to 2.4-nanometer-diamter SWNTs or DWNTs extending 50 to $750 \mathrm{~nm}$ beyond the end of the probe handle, the shortest and widest of which still have an aspect ratio comparable to the slenderest CNT AFM probes reported in the literature.

CM-AFM places substantially stricter requirements on the mechanical properties of the probe. Since the probe stays in contact with the surface as it is rastered, lateral as well as normal forces are applied to the probe. Continuous contact also results in much faster wear of the probe tip, especially when imaging high elastic modulus materials. Wear is expected to be a problem for any probe material; however, since CNTs have the highest elastic modulus of any known material they should be least susceptible to wear. Further, the pseudo-two-dimensional nature of the graphitic sheets that comprise the CNT surfaces generally present no asperities even on the atomic scale, making them even less susceptible to wear.

While the slenderness of CNTs is expected to provide reduced tip convolution in CM-AFM as it does in other methods, it is also expected to result in a more flexible probe - a source of concern because of the non-vanishing lateral forces applied to the probe tip during CMAFM. However, based on scanning anodic oxidation experiments, Dai et al. reported that flexure of the CNT is problematic only at scanning rates significantly faster than those generally used in CM-AFM, even for very slender CNT AFM probes, ${ }^{2}$ again due to the CNT's extremely high elastic modulus. While there is significant controversy regarding the bending rigidity of MWCNTs, ${ }^{34}$ recent theoretical studies suggest that for the same outer diameter, a greater number of walls does indeed increase bending stiffness. Further, unlike single-, and few-wall CNTs, which soften at relatively low strains due to large-scale buckling, more dense CNTs (i.e. CNTs with a greater number of walls), do not soften significantly at modest strains because the inner tubes resist buckling. We expect that by selecting denser MWCNTs, or CNT growth methods that favor denser MWCNTs, even slenderer CNT CM-AFM probes (or alternatively, faster scan rates) than those reported by Dai et al. can be realized.

CNT probes also have great potential for SPM-based nanoindentation. As in other scanning probe methods, the large aspect ratio gives CNT nanoindentation probes access to deep narrow recesses. Reproducible indentation measurements from the bottom of pits in the surface of a commercial polycarbonate DVD were reported by Akita and co-workers. ${ }^{41}, 42$ Further, the extremely high elastic modulus of CNTs makes them ideal for indentation applications. As in the case of CM-AFM, dense MWCNTs are expected to provide the best 
performance, since the presence of inner tubes reduces the compressive compliance, and increases flexural stiffness, thereby increasing the load necessary for Euler or shell buckling of the CNT.

Perhaps the most compelling benefit of using CNTs as nanoindentation probes, however, stems from the atomic-scale near-perfection of their tips. Traditional nanoindentation probes are made by mechanically grinding synthetic diamond. The grinding process yields a tip that is rough and irregular on the few-angstrom to few-nanometer scale. For extremely shallow indents, morphological irregularities result in a significant uncertainty in the projected area of the probe-sample contact area, complicating or even precluding quantitative analysis of the nanoindentation data. The smooth, hemispherical morphology of CNT tips, in conjunction with their high elastic modulus, is expected to reduce these uncertainties, possibly allowing quantitative determination of mechanical properties from few nanometer, or even sub-nanometer indents. The ability to probe mechanical properties to the sub-nanometer length scale would be of great benefit to the study of the tribological properties of industrially important thin films, such as gate insulators in VLSI circuits, and ultrathin diamond-like carbon films used in hard drives.

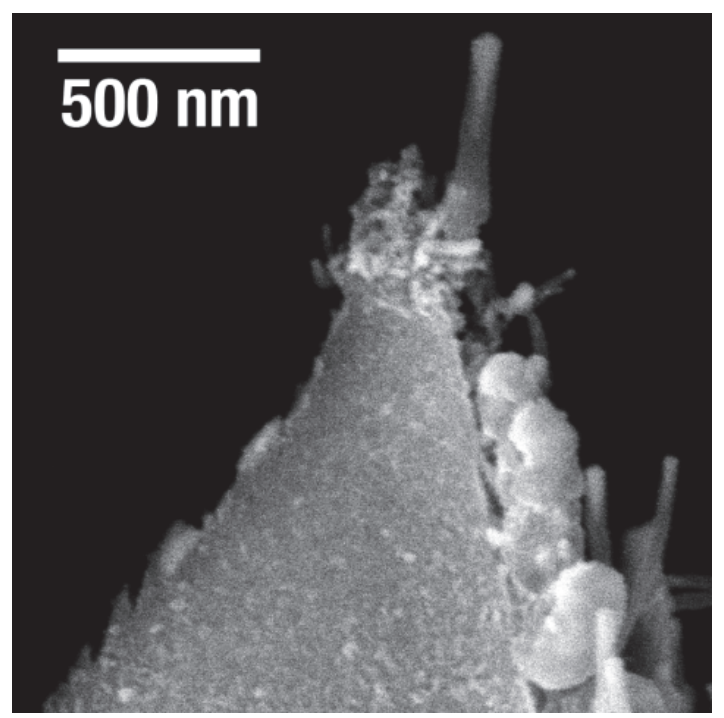

Fig. 4. SEM image of a single-CNT MFM probe fabricated using an ion-shadowed MPECVD fabrication method.

\subsection{Electronic considerations}

The electronic properties of CNTs generally make them a good probe material for SPM methods that require a conductive probe. CNTs are capable of carrying tremendous current densities. ${ }^{16}$ Several examples of CNT probes used successfully in EFM, scanning anodic oxidation lithography ${ }^{2}$ and nanoscale surface conductivity measurements. ${ }^{22,}{ }^{43}$ While it is well known that most synthetic methods produce $\sim 50 \%$ semiconducting, and further that even metallic CNTs generally have a very sparse density of states (DOS) near the (vacuum) Fermi level, empirically it is found that CNT SPM probes function very well for STM.1, 22, 44, 
45 The excellent performance of CNT SPM probes is somewhat confounding since materials with a sparse DOS near the Fermi level generally make poor SPM probes due to the small number of states available for electrons to tunnel out of and into. While further study is warranted, the absence of this problem in the case of CNT STM probes maybe attributed to a larger local DOS (LDOS) at the CNT tip due to broken bonds or the presence of the defects that are necessary to form the closed CNT tip. ${ }^{46}$ In some cases, residual metal catalyst particles at the probe tip may also be responsible for broadening the LDOS near the probe tip. .3

\section{CNT SPM probe fabrication methods}

Mounting a CNT - a definitively nanometer-scale object - on a millimeter-scale SPM probe (hereafter called the probe handle), is a non-trivial challenge. Approaches to this problem generally fall into one of two categories: direct attachment (DA), in which a previously grown CNT is attached to the tip of a SPM probe, and catalyst deposition / CVD methods (CDCVD), in which catalyst is deposited on a SPM probe and the CNTs are subsequently grown by CVD. Both categories have benefits and drawbacks. Modern DA methods are highly reproducible and offer good control of CNT position, angle, and length, but are tremendously time consuming. CDCVD methods are generally less reproducible, but much faster and less expensive. In the following, we describe the existing variations of DA methods, followed by a description of the existing methods of CDCVD methods. Each of these sections is organized roughly in order of increasing technical complexity, rather than chronologically. For completeness, we note that CNT SPM probe fabrication by magnetopheretic, ${ }^{47}$ and dielectropheretic ${ }^{48}$ attraction of solvated CNTs to the apex of the probe handle immersed in the CNT solution have also been reported; however, these methods have not been explored to the extent that CDCVD and DA methods have, being the subject of a single publication each. We thus, opt to direct interested readers to the pertinent references rather than describing them at length here.

\subsection{Probe fabrication by DA methods}

The first, and simplest DA CNT SPM probe fabrication method is that reported by Dai et al. in their seminal 1998 Science paper. ${ }^{1}$ In this method, bundles of arc-discharge-grown CNTs are secured to the probe handle with an acrylic adhesive. While the adhesion between the bundles and the probe handle has not, to our knowledge, been studied in detail, some authors suggest that sputtering Cr onto the CNT SPM probe strengthens the CNT bundle / probe handle junction. ${ }^{49}$ Optical microscopy is used to align the CNT bundles with probe handle during fabrication. Although the authors attest that in most cases one or two CNTs extends down below the rest of the bundle, this method does not produce single CNT probes.1, 2, 39, 49 In a similar approach, which does purportedly frequently yield single-CNT SPM probes, the probe handle is coated with a metal film to increase its conductivity, biased at $-5 \mathrm{~V} \sim-25 \mathrm{~V}$ relative to the CNT boule, and translated toward the CNT boule under the view of an optical microscope until a flash is observed. ${ }^{50}$ While the physics of this fabrication method was left largely unexplored, the authors suggest that electrostatic attraction is responsible for attracting the CNTs to the apex of the probe handle. The arc discharge evidenced by the flash is believed to follow the attached CNT burning through due to Joule heating at a defect within the CNT or at a CNT-CNT junction. This burning 
serves to sever the probe CNT from the boule, leaving, in some cases, an isolated CNT on the probe apex.

Alignment of CNTs with probe handle using microtranslators mounted in an scanning electron microscope (SEM) chamber, as first reported by Akita et al.,51, 52 is a greater technical challenge relative to the method of Dai et al., ${ }^{1}$ and is indeed more time consuming, but the results are significantly more consistent and reproducible. In the original report by Akita et al., CNTs were first dispersed in a solvent, and aligned perpendicular to the edge of a blade by dielectrophoresis. This provided a sparse array of highly aligned CNTs. The CNT-decorated blade and the probe handle were then mounted on separate translators inside the SEM chamber. The probe handle was aligned with, and then brought into contact with a CNT. Although the van der Waals force between the CNT and the probe handle is typically strong enough to hold the CNT in place, amorphous carbon is often deposited over the CNT/probe handle contact via electron-stimulated deposition by scanning the SEM's electron beam over a very small area around the junction. This method for amorphous carbon deposition requires a hydrocarbon background pressure of a few microtorr. Hydrocarbon contamination from a diffusion pump provides sufficient carbon feedstock, but carbon-containing molecules may have to be intentionally leaked into the chamber for the case of low-pressure turbo-molecular pump (TMP)-pumped chambers. The final step is generally to cut the CNT of the newly fabricated CNT SPM probe free of the blade by stopping the electron beam over the CNT. ${ }^{53}$ In a variant of this method used to make MFM probes, however, the probe is brought within one micrometer of the base of a CNT, and the CNT dislodged from the substrate and attached to the probe handle by applying a DC potential to the probe handle. ${ }^{54}$ This obviates the need for cutting of the CNT - an important consideration in the fabrication of MFM probes since the magnetic particle resides in the tip of the CNT. In spite of their inherent cost and complexity, SEM-based DA methods are currently the most reliable, reproducible and versatile method of CNT fabrication. In addition to AFM probes, $51-53,55$ and MFM probes, ${ }^{54}$ nanoindenter probes, $, 35,41,42$ four-point surface conductivity probes, ${ }^{22}$ and STM probes $^{45}$ have all been fabricated by various SEMbased DA methods.

\subsection{Probe fabrication by CDCVD methods}

Surprisingly, the simplest CDCVD CNT SPM probe fabrication method - simply depositing a layer of catalytically active metal on the probe handle and exposing to $\mathrm{CvD}$ conditions was among the last reported CDCVD methods. ${ }^{24,56,57}$ In the first report of simple CDCVD fabrication by Wongwiriyapan et al., the authors deposited $0.5 \mathrm{~nm} \sim 2 \mathrm{~nm}$ Fe films atop 5 $\mathrm{nm} \sim 10 \mathrm{~nm}$ Al buffer layers on W STM tips, and grew CNTs using thermal CVD with a $\mathrm{CH}_{4}$ carbon source. A significant drawback to thermal CVD is that the resulting CNTs are randomly oriented. Our group opted instead for microwave plasma enhanced CVD (MPECVD). ${ }^{24,43,57,58}$ In our MPECVD apparatus, a $200 \mathrm{~V}$ bias is applied between the sample holder and a second electrode held $\sim 10 \mathrm{~cm}$ above the sample holder (sample holder negative). The narrow velocity distribution of the ions that induce the CNT growth ensure that the CNTs grow toward the upper electrode with an angular distribution of approximately three degrees. ${ }^{59}$ Using MPECVD with a 15-nm Co catalyst films deposited on commercial AFM cantilevers, Tanaka et al. fabricated high-resolution MFM probes. ${ }^{24}$ Sakamoto et al. fabricated SPM probes decorated with Pd nanowire/MWCNT 
heterostructures using MPECVD with a thin Pd catalyst film deposited on AFM cantilevers. In both of the previously mentioned reports, the probe handle and the lower face of the cantilever were covered with an array of CNTs. Methods for tip-selective employing MPECVD will be described below.

Efforts to place the catalyst, and thus the subsequent CNTs, selectively at the tip of the probe handle by exploiting the electric field enhancement at the sharply curved tip were first reported by Hafner et $\mathrm{al}^{40}$ and Cheung et al. ${ }^{60}$ This method used dielectropherisis to attract Fe or FeMo impregnated alumina particles to the near-tip area of the probe handle, and subsequent thermal CVD with an ethylene feedstock for CNT growth. The resulting fewnanometere-diameter CNTs grew along the probe handle surface due to the strong van der Waals attraction, until they reached the probe apex. Since the energy to bend the CNT around the sharp tip of the probe handle exceeded the energy of the van der Waals attraction, the CNTs tended to extend out from the tip of the probe handle. Due to the nonoriented growth of thermal CVD, however, the length distribution of the CNT SPM probes was quite large, necessitating trimming. Tung, et al. exploited the enhanced electric field around the apex of a W STM tip to selectively electroplate Co catalyst near the apex.58 MPECVD growth yielded CNTs highly aligned along the long axis of the probe handle. Due to the controllable growth direction of the CNTs, CNT length can be controlled by adjusting growth time, and no trimming is necessary. The drawback of Tung's method (and all MPECVD-based methods) for some SPM applications is that MPECVD-grown CNTs tend to exhibit large diameters (15 nm 100 nm depending on catalyst size and chemical composition), compared to CNTs grown by thermal CVD.

A number of other methods for selectively depositing catalyst near the probe handle apex, and forcing the CNTs to grow in the desired direction during CVD have been reported. Charles Lieber's group at Harvard University developed and refined a method commonly known as pore growth wherein electrochemical etching was used to make pores in the tip of a Si AFM probe parallel to the long axis of the probe handle. Catalyst deposited in the pores yielded CNTs that were forced to grow along the pore direction during CVD growth. ${ }^{39,} 61,62$ For SSRM applications, we recently presented a method for the fabrication of nano-brushes of coaxial Pd nanowire/MWCNT heterostructures using a custom-fabricated area-selective electroplating cell to deposit Pd catalyst on the top $\sim 1 \mu \mathrm{m}$ of a commercial Si AFM probe, and the ion-induced alignment properties of MPECVD to force the Pd/MWCNTs to grow parallel with the probe handle long axis. ${ }^{43}$ More recently, we have developed a tip-selective variation on the method of Tanaka et al. described above. In this method, a FeNi alloy catalyst is deposited only on the forward face of a commercial Si AFM probe. The following MPECVD grows highly aligned, FeNi-nanoparticle-containing CNTs. While catalyst is deposited over a few square microns of the probe handle surface, the catalyst film at the probe handle apex blocks ions coming out of the plasma, preventing them from striking portions of the catalyst film away from the apex. This ion shadowing effect can be used to grow a single CNT at the probe handle apex with $\sim 70 \%$ reproducibility. The alignment and length of the CNT at the probe handle apex can be controlled purely by growth conditions. A representative probe of this type is shown in Fig. 3.

Because of the scalability of CDCVD methods a number of authors have attempted to develop mass-production CDCVD CNT SPM probe fabrication methods. Yenilmez et al. reported a method in which a wafer of silicon AFM probes was spin coated with a fewmicron layer of photoresist leaving only the pyramidal probe tips above the resist layer. 63 
Thereafter, a suspension of FeMo-impregnated alumina particles was spin coated on the wafer, the photoresist removed, and CNTs grown using thermal CVD with a methane carbon source. Unfortunately, this wafer-scale method produces CNTs that extend $1 \mu \mathrm{m} \sim 10 \mu \mathrm{m}$ beyond the end of the probe handle, necessitating that every CNT SPM probe be individually shortened by electrical discharge. ${ }^{1}$

\section{Summary and conclusions}

Due to their simple shape, high aspect ratio, exceptional mechanical and electronic properties CNTs have for many years been an extremely tempting material for scanning probe microscopists. Although the inherent complexities and uncertainties of CNT SPM probe fabrication have thus far precluded the sort of uniform wafer-scale production that would lead to affordable and readily available commercial CNT SPM probes, the 15 years since Dai et al.'s seminal paper, has seen the development of a wide array of serial, but extremely effective CNT SPM probe fabrication methods, and the application of these probes to wide array of SPM methods and a wide array of samples. In the preceding text, we have attempted to provide a one-stop guide to this body of work, in the hope of assisting future developers in their efforts to exploit the unique properties of CNTs in still more varieties of SPM. We expect that the coming years will see the variety of CNT SPM probes and the range of applications of CNT SPM probes expand even further.

\section{References}

[1] H. Dai, J. H. Hafner, A. G. Rinzler, D. T. Colbert and R. E. Smalley, Nature 384 (6605), 147-150 (1996).

[2] H. Dai, N. Franklin and J. Han, Applied Physics Letters 73 (11), 1508 (1998).

[3] C. A. Putman, K. O. v. d. Werf, B. G. d. Grooth, N. F. v. Hulst and J. Greve, Biophysical Journal 67 (4), 1749-1753 (1994).

[4] G. Binnig, H. Rohrer, C. Gerber and E. Weibel, Physical Review Letters 49 (1), 57-61 (1982).

[5] G. Binnig, H. Rohrer and C. Gerber, Physical Review Letters 56 (9), 930-933 (1986).

[6] G. Meyer and N. M. Amer, Applied Physics Letters 53 (12), 1045 (1988).

[7] F. J. Giessibl, Reviews of Modern Physics 75 (3), 949-983 (2003).

[8] Q. Zhong, D. Inniss, K. Kjoller and V. B. Elings, Surface Science Letters 290 (1-2), L688L692 (1993).

[9] Y. Martin and H. K. Wickramasinghe, Applied Physics Letters 50 (20), 1455-1457 (1987).

[10] J. J. Sáenz, N. García, P. Grütter, E. Meyer, H. Heinzelmann, R. Wiesendanger, L. Rosenthaler, H. R. Hidber and H. J. Güntherodt, Journal of Applied Physics 62 (10), 4293 (1987).

[11] U. Hartmann, Annual Review of Materials Science 29, 53-87 (1999).

[12] Y. Martin, D. W. Abraham and H. K. Wickramasinghe, Applied Physics Letters 52 (13), 1103 (1988).

[13] L. N. Kantorovich, A. I. Livshits and M. Stoneham, Journal of Physics: Condensed Matter 12 (6) (2000). 
[14] M. Nonnenmacher, M. P. O’Boyle and H. K. Wickramasinghe, Applied Physics Letters 58 (25), 2921- (1991).

[15] W. Melitza, J. Shena, A. C. Kummela and S. Lee, Surface Science Reports 66 (1), 1-27 (2011).

[16] M. S. Dresselhaus, G. Dresselhaus and P. C. Eklund, Science of fullerenes and carbon nanotubes. (Academic Press, 1996).

[17] M. Meyyappan, Journal of Physics D: Applied Physics 42, 213001 (2009).

[18] M. S. Arnold, A. A. Green, J. F. Hulvat, S. I. Stupp and M. C. Hersam, Nature Nanotechnology 1, 60 - 65 (2006).

[19] L. Jankovi, D. Gournis, P. N. Trikalitis, I. Arfaoui, T. Cren, P. Rudolf, M.-H. Sage, T. T. M. Palstra, B. Kooi, J. D. Hosson, M. A. Karakassides, K. Dimos, A. Moukarika and T. Bakas, Nano Letters 6 (6), 1131-1135 (2006).

[20] R. Li, X. Sun, X. Zhou, M. Cai and X. Sun, Journal of Physical Chemistry C 111 (26), 9130-9135 (2007).

[21] C. Müller, A. Leonhardt, M. C. Kutz and B. Büchner, Journal of Physical Chemistry C 113 (7), 2736-2740 (2009).

[22] S. Yoshimoto, Y. Murata, K. Kubo, K. Tomita, K. Motoyoshi, T. Kimura, H. Okino, R. Hobara, I. Matsuda, S.-i. Honda, M. Katayama and S. Hasegawa, Nano Letters 7 (4), 956-959 (2007).

[23] Z. Deng, E. Yenilmez, J. Leu, J. E. Hoffman, E. W. J. Straver, H. Dai and K. A. Moler, Applied Physics Letters 85 (25), 6263-6265 (2004).

[24] K. Tanaka, M. Yoshimura and K. Ueda, Journal of Nanomaterials 2009, 147204 (2009).

[25] I. T. Clark, Y. Matsuoka and M. Yoshimura, in preperation.

[26] C. M. Wang, Y. Y. Zhang, Y. Xiang and J. N. Reddy, Applied Mechanics Reviews 63 (3), 030804 (2010).

[27] M. M. Shokrieh and R. Rafiee, Mechanics of Composite Materials (2010).

[28] O. Lourie, D. M. Cox and H. D. Wagner, Physical Review Letters 81 (8), 1638-1641 (1998).

[29] J. F. Waters, L. Riester, M. Jouzi, P. R. Guduru and J. M. Xu, Applied Physics Letters 85 (10), 1787-1789 (2004).

[30] J. F. Waters, P. R. Guduru, M. Jouzi, J. M. Xu, T. Hanlon and S. Suresh, Applied Physics Letters 87 (10), 103109 (2005).

[31] P. Zhang, P. E. Lammert and V. H. Crespi, Physical Review Letters 81 (24), 5346-5349 (1998).

[32] D. Srivastava, M. Menon and K. Cho, Physical Review Letters 83 (15), 2973-2976 (1999).

[33] M. R. Falvo, G. J. Clary, R. M. Taylor, V. Chi, J. F.P. Brooks, S. Washburn and R. Superfine, Nature 389, 582-584 (1997).

[34] P. Poncharal, Z. L. Wang, D. Ugarte and W. A. d. Heer, Science 283, 1513-1516 (1999).

[35] S. AKITA, H. NISHIJIMA, T. KISHIDA and Y. NAKAYAMA, Japanese Journal of Applied Physics 39, 3724-3727 (2000).

[36] S. D. Solares, Y. Matsuda and W. A. G. III, Journal of Physical Chemistry B 109, 1665816664 (2005).

[37] Y.-R. Jeng, P.-C. Tsai and T.-H. Fang, Applied Physics Letters 90 (16), 161913 (2007).

[38] X. Duan, C. Tang, J. Zhang, W. Guo and Z. Liu, Nano Letters 7 (1), 143-148 (2007). 
[39] S. S. Wong, J. D. Harper, P. T. Lansbury and C. M. Lieber, Journal of the American Chemical Society 120, 603 (1998).

[40] J. H. Hafner, C. L. Cheung and C. M. Lieber, Journal of the American Chemical Society 121 (41), 9750-9751 (1999).

[41] S. Akita, H. Nishijima, T. Kishida and Y. Nakayama, Japanese Journal of Applied Physics 39, 7086-7089 (2000).

[42] S. Akita and Y. Nakayama, Japanese Journal of Applied Physics 40, 4289-4291 (2001).

[43] I. T. Clark, G. Rius, Y. Matsuoka and M. Yoshimura, Journal of Vacuum Science and Technology B 28 (6), 1148-1152 (2010).

[44] A. Pasquini, G. B. Picotto and M. Pisani, Sensors and Actuators A 123-124, 655-659 (2005).

[45] N. Isomura, X. Wu and Y. Watanabe, Journal of Chemical Physics 131, 164707 (2009).

[46] A. D. Vita, J.-C. Charlier, X. Blase and R. Car, Applied Physics A 68, 283-286 (1999).

[47] A. Hall, W. G. Matthews, R. Superfine, M. R. Falvo and S. Washburn, Applied Physics Letters 82 (15), 2506-2508 (2003).

[48] H. W. Lee, S. H. Kim and Y. K. Kwak, Reviews of Scientific Instruments 76, 046108 (2005).

[49] J. Li, A. M. Cassell and H. Dai, Surface and Interface Analysis 28, 8-11 (1999).

[50] R. M. D. Stevens, N. A. Frederick, B. L. Smith, D. E. Morse, G. D. Stucky and P. K. Hansma, Nanotechnology 11, 1-5 (2000).

[51] S. Akita, H. Nishijima, Y. Nakayama, F. Tokumasu and K.Takeyasu, Journal of Physics D: Applied Physics 32, 1044-1048 (1999).

[52] H. Nishijima, S. Kamo, S. Akita, Y. Nakayama, K. I. Hohmura, S. H. Yoshimura and K.Takeyasu, Applied Physics Letters 74, 4061-4063 (1999).

[53] J. Martinez, T. D. Yuzvinsky, A. M. Fennimore, A. Zettl, R. Garcia and C. Bustamante, Nanotechnology 16, 2493-2496 (2005).

[54] T. Arie, H. Nishijima, S. Akita and Y. Nakayama, Journal of Vacuum Science and Technology B 18 (1), 104-106 (2000).

[55] K. I. Hohmura, Y. Itokazu, S. H. Yoshimura, G. Mizuguchi, Y.-s. Masamura, K. Takeyasu, Y. Shiomi, T. Tsurimoto, H. Nishijima, S. Akita and Y. Nakayama, Journal of Electron Microscopy 49 (3), 415-421 (2000).

[56] W. Wongwiriyapan, S.-i. Honda, T. Mizuta, T. Ohmori, T. Murakami, K. Kisoda, H. Harima, J.-G. Lee, H. Mori, K. Oura and M. Katayama, Japanese Journal of Applied Physics 45 (3A), 1880-1882 (2006).

[57] T. Sakamoto, C.-C. Chiu, K. Tanaka, M. Yoshimura and K. Ueda, Journal of Nanomaterials 2009, 851290 (2009).

[58] F.-K. Tung, M. Yoshimura and K. Ueda, Journal of Nanomaterials 2009, 612549 (2009).

[59] I. T. Clark, Y. Matsuoka and M. Yoshimura, in preperation.

[60] C. L. Cheung, J. H. Hafner, T. W. Odom, K. Kim and C. M. Lieber, Applied Physics Letters 76 (21), 3136-3138 (2000).

[61] C. L. Cheung, J. H. Hafner and C. M. Lieber, PNAS 97 (8), 3809-3813 (2000). 
[62] A. T. Woolley, C. Y. Cheung, J. H. Hafner and C. M. Lieber, Chemistry and Biology 7, R193-R204 (2000).

[63] E. Yenilmez, Q. Wang, R. J. Chen, D. Wang and H. Dai, Applied Physics Letters 80 (12) (2002). 


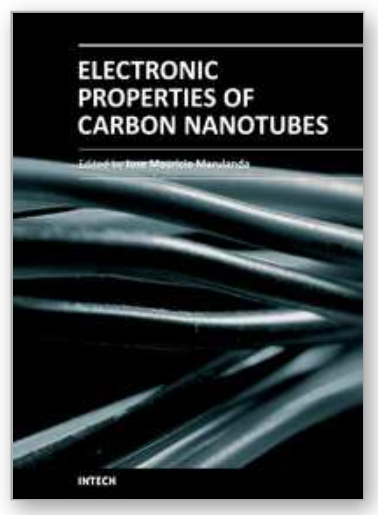

\author{
Electronic Properties of Carbon Nanotubes \\ Edited by Prof. Jose Mauricio Marulanda
}

ISBN 978-953-307-499-3

Hard cover, 680 pages

Publisher InTech

Published online 27, July, 2011

Published in print edition July, 2011

Carbon nanotubes (CNTs), discovered in 1991, have been a subject of intensive research for a wide range of applications. These one-dimensional (1D) graphene sheets rolled into a tubular form have been the target of many researchers around the world. This book concentrates on the semiconductor physics of carbon nanotubes, it brings unique insight into the phenomena encountered in the electronic structure when operating with carbon nanotubes. This book also presents to reader useful information on the fabrication and applications of these outstanding materials. The main objective of this book is to give in-depth understanding of the physics and electronic structure of carbon nanotubes. Readers of this book should have a strong background on physical electronics and semiconductor device physics. This book first discusses fabrication techniques followed by an analysis on the physical properties of carbon nanotubes, including density of states and electronic structures. Ultimately, the book pursues a significant amount of work in the industry applications of carbon nanotubes.

\title{
How to reference
}

In order to correctly reference this scholarly work, feel free to copy and paste the following:

Ian Thomas Clark and Masamichi Yoshimura (2011). Fabrication of Carbon Nanotubes for High-Performance Scanning Probe Microscopy, Electronic Properties of Carbon Nanotubes, Prof. Jose Mauricio Marulanda (Ed.), ISBN: 978-953-307-499-3, InTech, Available from: http://www.intechopen.com/books/electronic-properties-ofcarbon-nanotubes/fabrication-of-carbon-nanotubes-for-high-performance-scanning-probe-microscopy

\section{INTECH}

open science | open minds

\section{InTech Europe}

University Campus STeP Ri

Slavka Krautzeka 83/A

51000 Rijeka, Croatia

Phone: +385 (51) 770447

Fax: +385 (51) 686166

www.intechopen.com

\section{InTech China}

Unit 405, Office Block, Hotel Equatorial Shanghai

No.65, Yan An Road (West), Shanghai, 200040, China

中国上海市延安西路65号上海国际贵都大饭店办公楼 405 单元

Phone: +86-21-62489820

Fax: $+86-21-62489821$ 
(C) 2011 The Author(s). Licensee IntechOpen. This chapter is distributed under the terms of the Creative Commons Attribution-NonCommercialShareAlike-3.0 License, which permits use, distribution and reproduction for non-commercial purposes, provided the original is properly cited and derivative works building on this content are distributed under the same license. 